\title{
Millimeter/Submillimeter Mixing Based on the Nonlinear Plasmon Response of Two-Dimensional Electron Systems
}

\author{
V. M. Muravev ${ }^{a, b}$, I. V. Kukushkin ${ }^{a, b}$, J. Smet ${ }^{b}$, and K. von Klitzing ${ }^{b}$ \\ ${ }^{a}$ Institute of Solid State Physics, Russian Academy of Sciences, Chernogolovka, Moscow region, 142432 Russia \\ ${ }^{b}$ Max-Planck-Institut für Festkörperforschung, Heisenbergstrasse 1, D-70569 Stuttgart, Germany
}

Received June 25, 2009

\begin{abstract}
The nonlinear plasmon response of a two-dimensional electron system with an incorporated defect to monochromatic and bichromatic microwave radiation has been investigated. The operation of an electronic device (mixer) based on the plasmon response with a record operational speed has been demonstrated and analyzed. It has been found that the system response time is no more than $\tau=25 \mathrm{ps}$. It has been shown that the nonlinear response of the system is caused by a new physical mechanism of nonlinearity induced by the presence of an inhomogeneity in the electron system.
\end{abstract}

PACS numbers: 73.23.-b

DOI: $10.1134 / \mathrm{S} 0021364009150089$

In recent years, the millimeter/submillimeter wavelength range (terahertz radiation) has attracted the increased interest of researchers [1]. This interest is partially explained by a number of surprising properties of radiation of this range. Terahertz radiation penetrates without noticeable damping through a number of subjects nontransparent for visible light and does not have an ionizing action detrimental to living systems. A number of chemical reagents have unique spectral features in this range, which can be used to recognize substances. Moreover, the investigations of space submillimeter radiation make it possible to obtain much information on the content, origin, and evolution of the galaxies of the universe. The insufficient technological capability of this range hinders the active development of investigations and applications in the terahertz range. Cheap miniature tunable detectors and oscillators are almost absent in the terahertz frequency range, because the physical principles underlying the operation of microwave and light devices become invalid in this wavelength range. The aim of this work is to propose and test a new physical principle for creating electronic devices, which can be widely applied in the detection systems for millimeter/submillimeter radiation.

Heterodyne detectors are the most widely used receivers of terahertz radiation. The operation principle of heterodyne detectors is that the electromagnetic radiation from a subject under investigation is mixed with radiation of a known frequency from a heterodyne source and is directed to a nonlinear element. A signal at the intermediate frequency $\Delta f$, which is the difference between the frequencies of radiation from the heterodyne source and the object under investigation, is detected on the nonlinear element. Thus, the spectroscopy of radiation emitted by the object under investigation is performed. A Schottky diode [2] is the most widespread nonlinear element of the heterodyne schemes. Its operation is based on the nonlinearity of the current-voltage characteristic of the Schottky junction (metal-semiconductor junction). The disadvantages of the heterodyne schemes based on the Schottky diode are a narrow bandwidth $(1 \mathrm{GHz})$ and a high absorbing power from the heterodyne source $(1 \mathrm{~mW})$. Mixers based on a superconductor-insulator-superconductor junction (SIS mixers) constitute another widespread class of heterodyne detectors [3]. This class of mixers has record sensitivity and noise temperature parameters. The principle of their operation is based on the strongly nonlinear behavior of the current-voltage characteristic of the SIS junction at voltages at which the tunneling of Cooper pairs from one superconductor to another begins. The main disadvantage of this class of mixers is a very low working temperature of about $4.2 \mathrm{~K}$, which strongly complicates working with them. Bolometers constitute the third type of nonlinear elements used in the heterodyne schemes. The mechanism of the operation of bolometers is based on the heating of the electron subsystem of the structure under the absorption of electromagnetic radiation. The heating of the electron subsystem changes the sample resistivity at the intermediate frequency $\Delta f$. The flow of a direct current through the sample is accompanied by the appearance of a photovoltage signal, which is directly detected. The transmission band of bolometric mixers is determined by the energy relaxation time of hot charge carriers. Mixers based on the heating effect in a thin superconductor film [4] are typical heterodyne detectors. These heterodyne detectors have record parame- 


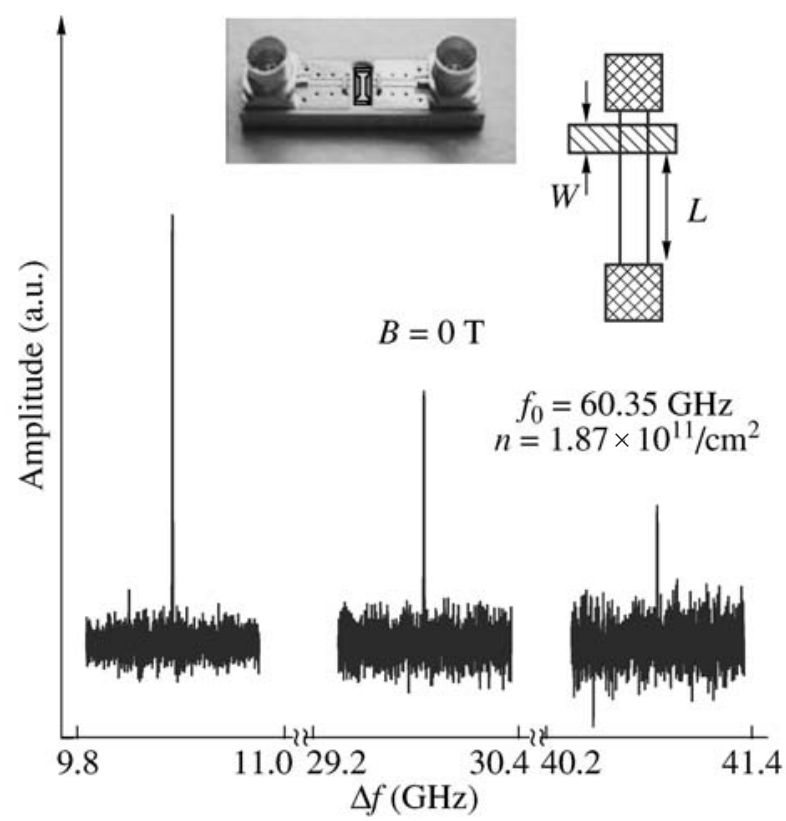

Fig. 1. Spectra of a signal from a nonlinear element at three intermediate frequencies $\Delta f=10.35,29.83$, and $40.82 \mathrm{GHz}$. The frequency of the heterodyne radiation source was $f_{0}=60.35 \mathrm{GHz}$ and the output power was $10 \mathrm{~mW}$. The radiation power from the signal generator was lower than the power from the heterodyne generator by $10 \mathrm{~dB}$. The sample temperature was $1.5 \mathrm{~K}$. The scheme of the structure under investigation and the photo of a strip line with the sample at its end are given in the upper part of the figure.

ters of sensitivity and noise temperature owing to a very steep temperature dependence of electric resistivity near the superconducting transition. However, owing to the inertness of the cooling of hot charge carriers through the interaction with phonons or diffusion cooling, the device transmission band is no wider than $10 \mathrm{GHz}$. Mixers on a two-dimensional electron gas in AlGaAs/GaAs semiconductor heterostructures [5, 6] constitute an alternative type of bolometric heterodyne detectors operating at temperatures of up to $80 \mathrm{~K}$. The maximum attainable transmission band for the case of the cooling of hot charge carriers on the optical phonons of the lattice is $4 \mathrm{GHz}$ [5-7]. The transmission bands for the cases of the cooling of hot charge carriers owing to their diffusion and ballistic emission to the contacts are 20 [8] and $40 \mathrm{GHz}$ [9], respectively. However, the noise temperature and power consumed from the heterodyne source for $\mathrm{AlGaAs} / \mathrm{GaAs}$ mixers are much higher than those for superconductor analogs and are 1000-2000 K and $0.5-1 \mu \mathrm{W}$, respectively. It is also worth noting a promising proposal of using a short carbon nanotube as a nonlinear bolometric element of a mixer [10, 11]. Since the Fermi velocity in the carbon nanotubes is an order of magnitude higher than that in $\mathrm{AlGaAs} / \mathrm{GaAs}$ heterostructures, the transmission band of the mixer in the regime of the ballistic cooling of hot charge carriers can reach hundreds of gigahertz.

The operation of all of the above mixers is based on the nonlinearity in the drift of the charge carriers. For this reason, the response time of a device $\tau$ even in the ballistic regime is limited by the time $L / V$, where $L$ is the size of the nonlinear element of the mixer ( $L$ is usually no less than $1 \mu \mathrm{m}$ ) and $v$ is the typical velocity of charge carriers in the device (usually, $v$ is about the Fermi velocity and is no more than $10^{7} \mathrm{~cm} / \mathrm{s}$ ). Thus, the response time of the device is fundamentally limited by the time $\tau \sim 10^{-11} \mathrm{~s}$ and the transmission band of the device is limited by a frequency of $100 \mathrm{GHz}$. One of the possibilities of decreasing the response time of modern electronic devices is the use of plasma waves as carriers of electric signals. Indeed, the velocity of plasma excitations in two-dimensional electron systems can reach $v_{p}=10^{9} \mathrm{~cm} / \mathrm{s}[12,13]$, which is two orders of magnitude higher than the maximally attainable electron drift velocity. This can reduce the response time of devices to $\tau \sim 10^{-13} \mathrm{~s}$ and increase the transmission band to a frequency of $10 \mathrm{THz}(L=$ $1 \mu \mathrm{m})$. The first attempts to use plasma waves as fast carriers of electric signals were made in $[14,15]$, where the transmission band of a mixer based on a fieldeffect transistor with a lattice gate was investigated. However, the nonlinear response of the transistor at the intermediate frequency appeared to be caused by the bolometric heating of the electron system, which prevented the reaching of the desired response time of the system. In this work, the operation of an electronic device based on the plasmon response with a record operational speed is demonstrated and analyzed. The system response time is no more than $\tau=25 \mathrm{ps}$. The nonlinear response of the two-dimensional electron system with an incorporated defect to monochromatic and bichromatic microwave radiation is experimentally studied. It is shown that the nonlinear response of the system is caused by a new physical mechanism of nonlinearity induced by the presence of an inhomogeneity in the electron system.

Investigations were performed on a single GaAs/AlGaAs heterojunction located at a depth of $190 \mathrm{~nm}$ from the structure surface. Two types of the structures with the electron densities $n_{s}=1.87 \times 10^{11}$ and $3.5 \times 10^{11} \mathrm{~cm}^{-2}$ were used in the investigations. The electron mobility at a temperature of $4.2 \mathrm{~K}$ was no less than $10^{6} \mathrm{~cm}^{2} /(\mathrm{Vs})$. A mesa, which is a strip of twodimensional electrons with two $\mathrm{Au} / \mathrm{Ge}$ contacts at the ends and a metallic gate sputtered on the crystal surface, was produced on the structure by means of lithography. Figure 1 shows the scheme of the structure. The width of the two-dimensional electron strip was $50 \mu \mathrm{m}$ and its total length was $275 \mu \mathrm{m}$. The distance from the lower contact to the gate was $L=$ $200 \mu \mathrm{m}$ and the gate width was $W=50 \mu \mathrm{m}$. The sample fixed at the end of a $16-\mathrm{mm}$ waveguide was placed into a helium cryostat inside a superconducting sole- 
noid. Mixed microwave radiation from the signal and heterodyne generators was supplied to the sample through a waveguide. The power of the heterodyne generator in the experiments was higher than the power of the signal generator by $10 \mathrm{~dB}$. Microwave generators cover a frequency range from 1 to $80 \mathrm{GHz}$ at the output power of no higher than $10 \mathrm{~mW}$. The sample was placed at the end of the stripline matched with a coaxial cable (see Fig. 1). Radiation at the intermediate frequency from the sample passed through the stripline and coaxial cable to the input of a spectrograph. The input frequency band of the spectrograph was $40 \mathrm{GHz}$.

Figure 1 shows the spectra of a signal from a nonlinear element at three intermediate frequencies $\Delta f=$ $10.35,29.83$, and $40.82 \mathrm{GHz}$. The frequency of the heterodyne source was $f_{0}=60.35 \mathrm{GHz}$, its output power was $10 \mathrm{~mW}$, and the output power of the signal generator was $1 \mathrm{~mW}$. The magnetic field was chosen so as to obtain the maximum signal and was $B=0 \mathrm{~T}$ in this experiment. It is seen that the amplitude of the signal from the mixer decreases by a factor of only one fourth when the intermediate frequency increases up to $40 \mathrm{GHz}$ (the instrumental limit for the used spectrograph). Thus, at a temperature of $T=1.5 \mathrm{~K}$, the transmission band of the mixer under investigation is no less than $40 \mathrm{GHz}$; correspondingly, the response time of the nonlinear element of the mixer is no more than $\tau=25 \mathrm{ps}$. This system response time at a nonlinear element size of $L=0.2 \mathrm{~mm}$ indicates that perturbation propagates in the system with a velocity of no less than $V_{\mathrm{p}}=0.8 \times 10^{9} \mathrm{~cm} / \mathrm{s}$. This velocity is the record value experimentally determined for the receivers of millimeter/submillimeter radiation. A two-dimensional electron system with an incorporated defect is the nonlinear element of the mixer under investigation. A plasma wave (plasmon) is the only excitation that can propagate in the two-dimensional electron system with such a velocity. Plasma waves in the two-dimensional electron system are waves generated by the Coulomb interaction between fluctuations of the charge density of the system.

To test the plasmon mechanism of the response of the system under investigation and to determine the role of the defect in the formation of its nonlinear properties, we carried out magnetic field measurements. The upper line in Fig. 2 corresponds to the magnetic field dependence of the amplitude of the signal from the nonlinear element at the intermediate frequency $\Delta f=0.9 \mathrm{GHz}$. The frequency of the heterodyne source was $f_{0}=48.32 \mathrm{GHz}$. The lower line in this figure is the magnetic field dependence of the photovoltage signal measured between a pair of contacts of the same nonlinear element under monochromatic irradiation at the frequency $f_{0}=48.32 \mathrm{GHz}$. Magnetic field oscillations in the photovoltage signal are caused by the interference of edge magnetoplasma waves at the size $L=0.2 \mathrm{~mm}$ equal to the distance between the

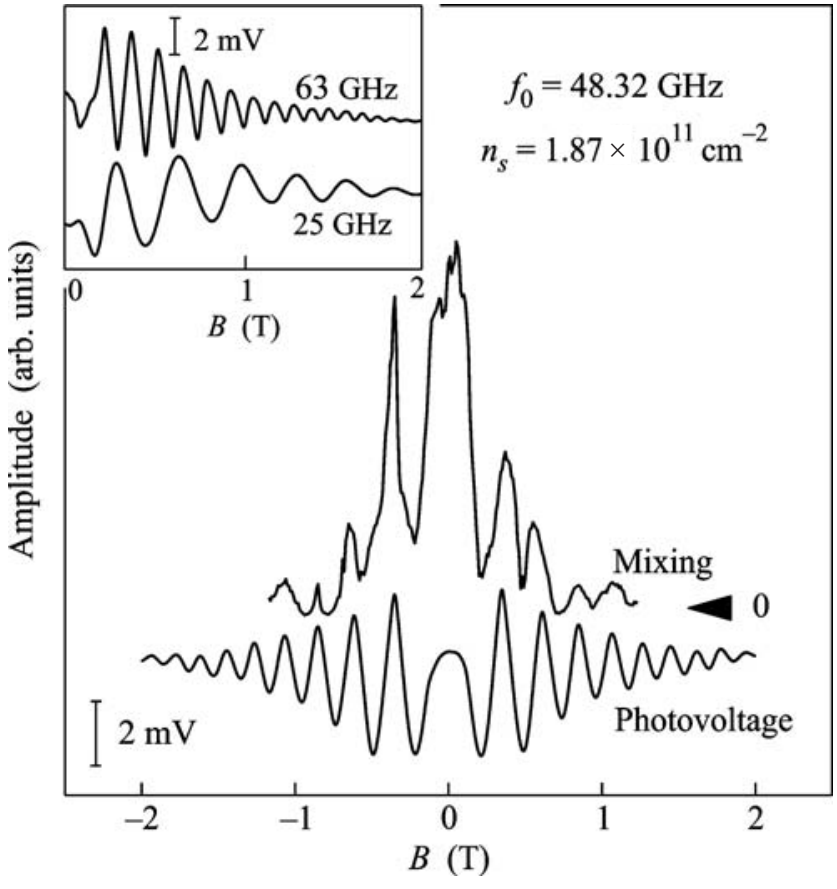

Fig. 2. Magnetic field dependences of the signal at the intermediate frequency $\Delta f=0.9 \mathrm{GHz}$ and the photovoltage measured from a pair of the contacts of the sample under investigation. The arrow marks the zero level of the mixing signal. The frequency of the heterodyne radiation source was $f_{0}=48.32 \mathrm{GHz}$. It is seen that the maxima and minima in the signal at the intermediate frequency coincide with the respective maxima and minima in the photovoltage. This indicates that the system response at the intermediate frequency is caused by the plasmon nonlinearity mechanism. The inset shows the magnetic field oscillations in the photovoltage for two microwave radiation frequencies, 25 and $63 \mathrm{GHz}$.

defect and contact to the two-dimensional electron system (see Fig. 1) [16-18]. As shown below, the photovoltage signal appears due to the rectification of the alternating potential of a magnetoplasma wave on the system nonlinearity caused by the presence of the inhomogeneity of the electron density (defect) in the system. The frequency of the magnetoplasma wave coincides with the frequency of the heterodyne source. For the experimental test of the interference nature of the observed photovoltage oscillations, we performed frequency measurements. The inset in Fig. 2 shows the photovoltage oscillations measured for two microwave radiation frequencies 25 and $63 \mathrm{GHz}$. It is seen that the oscillation period is inversely proportional to the radiation frequency. Indeed, under the constructive interference of edge magnetoplasma waves at the distance $L$, the relation $q L=2 \pi N$ is satisfied, where $q$ is the wave vector of the magnetoplasmon and $N=1,2$, 3 is the interference order. The dispersion of the edge magnetoplasma waves is described by the expression $\omega \propto n_{s} q / B$ [19]. Therefore, the successive magnetoplasma interference maxima are spaced by the same 


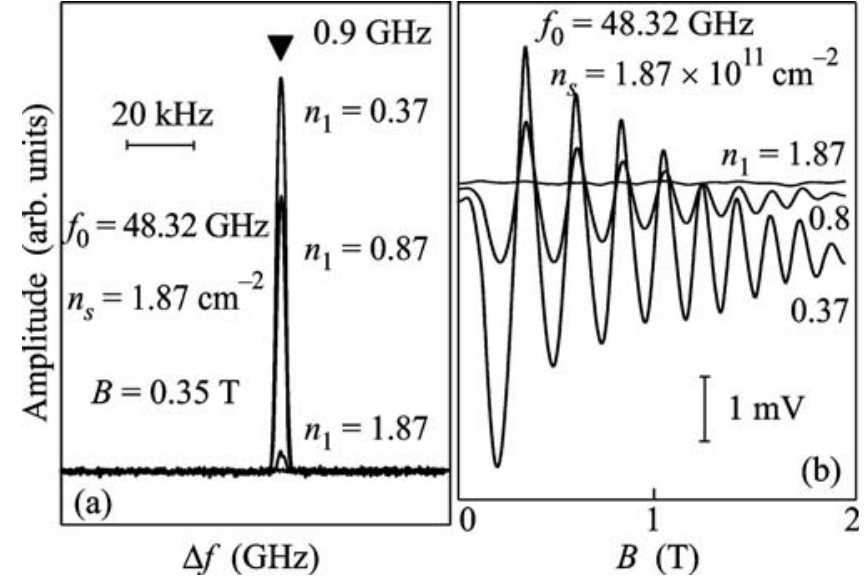

Fig. 3. (a) System response at the intermediate frequency $\Delta f=0.9 \mathrm{GHz}$ versus the electron density $n_{1}$ in the defect region. The electron density far from the defect is $n_{s}=$ $1.87 \times 10^{11} \mathrm{~cm}^{-2}$. The system response increases strongly under the depletion of the subgate region of the structure. (b) Magnetic field oscillations of the photovoltage at various electron densities $n_{1}$ in the defect region. The frequency of microwave radiation irradiating the sample is $f_{0}=48.32 \mathrm{GHz}$. All dependences were obtained at a sample temperature of $1.5 \mathrm{~K}$.

magnetic field distance $\Delta B \propto n_{s} / \omega$, which is in good agreement with the experimental results. The maxima and minima in the photovoltage as a function of the magnetic field coincide with the respective maxima and minima in the intermediate frequency signal. This coincidence certainly confirms that the system response at the intermediate frequency is determined by the behavior of plasma waves in the system under study. Figure 3a shows the dependence of the amplitude of the signal from the nonlinear element at the intermediate frequency on the electron density in the defect region $n_{1}$. The signal was measured at the intermediate frequency $\Delta f=0.9 \mathrm{GHz}$ at the plasmon interference maximum as a function of the magnetic field $B=0.35 \mathrm{~T}$. The electron density $n_{1}$ in the defect region was varied by supplying a voltage to a gate (see Fig. 1). It is seen that an increase in the inhomogeneity of the electron density leads to an increase in the nonlinearity of the system, resulting in an increase in the signal. The individual experiments show that the nonlinear response of the two-dimensional electron strip without a defect is negligibly small. Similar results were obtained for the magnetic field oscillations of the photovoltage (see Fig. $3 b$ ). When the electron density jump $n-n_{1}$ increases from 0 to $1.5 \times 10^{11} \mathrm{~cm}^{-2}$, the oscillation amplitude increases by a factor of 100 . Moreover, it was found that the observed plasmon nonlinearity can be caused by any inhomogeneity in the physical properties and topology of the electron system, as well as in its dielectric environment [20]. The physical mechanism responsible for the appear-

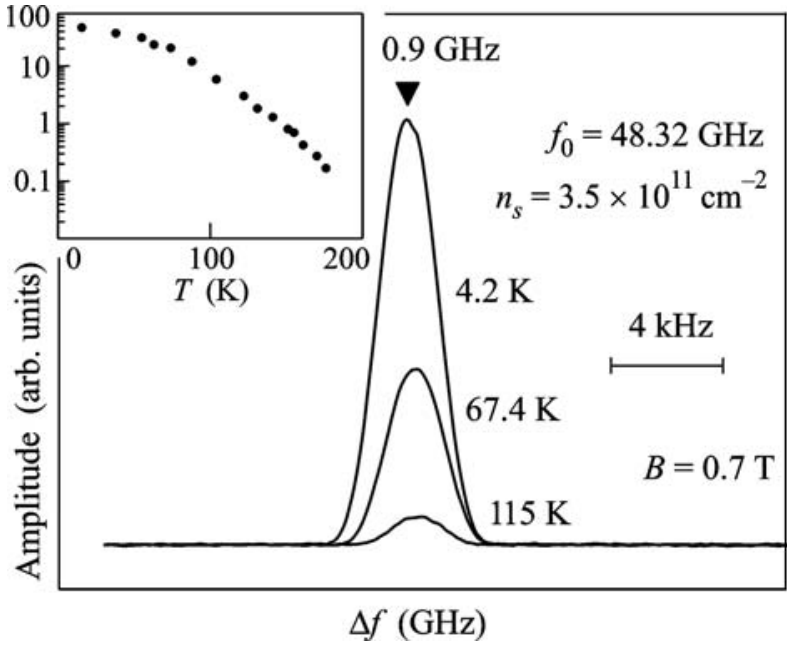

Fig. 4. Spectra of the signal from the nonlinear element at three temperatures $T=4.2,67.4$, and $115 \mathrm{~K}$. The frequency of the heterodyne radiation source was $f_{0}=$ $60.35 \mathrm{GHz}$. The measurements were performed at the intermediate frequency $\Delta f=0.9 \mathrm{GHz}$ at the maximum of the plasmon interference in the magnetic field $B=0.7 \mathrm{~T}$. The inset shows a more detailed temperature dependence of the signal amplitude.

ance of the plasmon nonlinearity on such defects is under investigation now and will be reported later.

Figure 4 illustrates the temperature dependence of the effect. The signal was measured at the intermediate frequency $\Delta f=0.9 \mathrm{GHz}$ at the plasma interference maximum as a function of the magnetic field $B=$ $0.7 \mathrm{~T}$. The two-dimensional electron density in the structure under investigation was $3.5 \times 10^{11} \mathrm{~cm}^{-2}$. Three spectra from the nonlinear element at temperatures of 4.2, 67.4, and $115 \mathrm{~K}$ are shown. It is seen that the signal decreases only by an order of magnitude when the temperature is increased from 4.2 to $100 \mathrm{~K}$. The inset in Fig. 4 shows a more detailed temperature dependence of the signal amplitude. The temperature behavior of the effect is explained by two factors. The first factor is the temperature dependence of the amplitude of the alternating potential of the plasma waves interfering inside the structure [17]. The second factor is the temperature behavior of the nonlinearity of the electron system. The experiments show that the first and second factors dominate at temperatures below $120 \mathrm{~K}$ and above $150 \mathrm{~K}$, respectively.

Figure 5 illustrates a change in the bandwidth of the nonlinear element with increasing temperature. The results are the generalization of the data presented in Fig. 1 to the case of different temperatures. The frequency of the heterodyne source was $f_{0}=60.35 \mathrm{GHz}$, its output power was $10 \mathrm{~mW}$, and the output power of the signal generator was $1 \mathrm{~mW}$. The magnetic field was chosen in order to obtain the maximum signal and was $B=0 \mathrm{~T}$ in this experiment. The transmission band at 


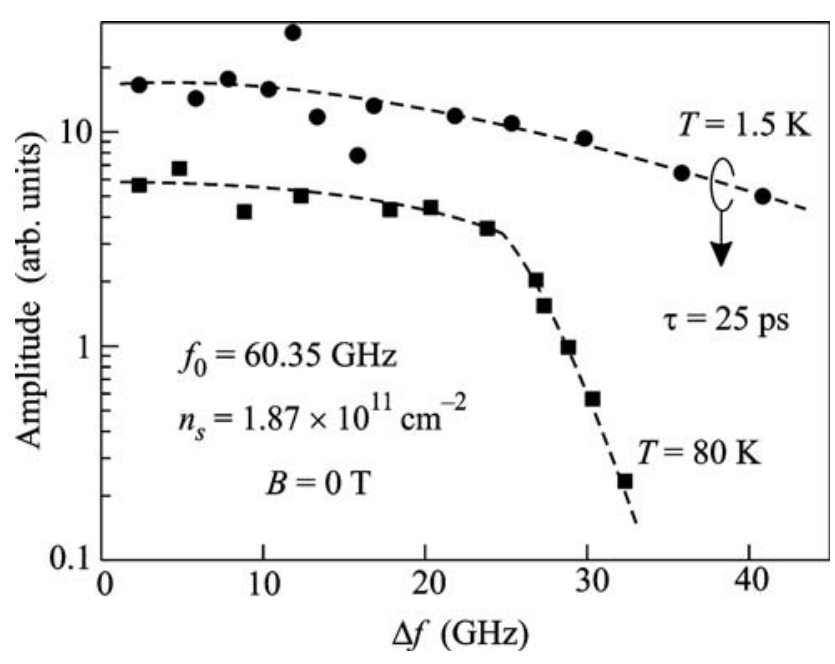

Fig. 5. Illustration of a change in the bandwidth of the nonlinear plasmon element with increasing temperature. The transmission band of the system under consideration at the temperature $T=1.5 \mathrm{~K}$ is no less than $40 \mathrm{GHz}$, which corresponds to the system response time of no more than $\tau=25 \mathrm{ps}$. When the temperature increases to $80 \mathrm{~K}$, a sharp cutoff of the mixing efficiency is observed and the band decreases to $27 \mathrm{GHz}$.

the temperature $T=1.5 \mathrm{~K}$ is no less than $40 \mathrm{GHz}$, which corresponds to the system response time of no more than $\tau=25$ ps. When the temperature is increased to $T=80 \mathrm{~K}$, a sharp cutoff of the mixing efficiency is observed and the band decreases to 27 GHz. This result is likely due to the fact that the highfrequency properties of the defect begin to affect the behavior of the system with increasing temperature.

To summarize, the nonlinear plasmon response of the two-dimensional electron system with the incorporated defect to monochromatic and bichromatic microwave radiation has been investigated in this work. The operation of an electronic device (mixer) based on the plasmon response with a record operational speed has been demonstrated and analyzed. It has been found that the system response time is no more than $\tau=25 \mathrm{ps}$. It has been shown that the nonlinear response of the system is caused by a new physical mechanism of nonlinearity induced by the presence of an inhomogeneity in the electron system.

We are grateful to V.A. Volkov for stimulating discussions and remarks. This work was supported by the Russian Foundation for Basic Research and the Fed- eral Agency for Science and Innovations. V.M.M. also acknowledges the support of the Landau Scholarship (Forschungzentrum, Jülich, Germany) and the Foundation for the Promotion of the Development of Small Enterprises.

\section{REFERENCES}

1. M. Tonouchi, Nature Photon. 1, 97 (2007).

2. T. W. Crowe, R. J. Mattauch, H. P. Roser, et al., Proc. IEEE 80, 1827 (1992).

3. M. J. Wengler, Proc. IEEE 80, 1810 (1992).

4. E. M. Gershenzon, G. N. Gol'tsman, I. G. Gogidze, et al., Sov. Phys. Supercond. 3, 1582 (1990).

5. J. X. Yang, F. Agahi, D. Dai, et al., IEEE Trans. Microwave Theory Tech. 41, 581 (1993).

6. J. X. Yang, J. Li, C. F. Musante, et al., Appl. Phys. Lett. 66, 1983 (1995).

7. D. V. Morozov, K. V. Smirnov, A. V. Smirnov, et al., Fiz. Tekh. Poluprovodn. 39, 1117 (2005).

8. M. Lee, L. N. Pfeiffer, K. W. West, et al., Appl. Phys. Lett. 78, 2888 (2001).

9. M. Lee, L. N. Pfeiffer, K. W. West, et al., Appl. Phys. Lett. 81, 1243 (2002).

10. K. S. Yngvesson, Appl. Phys. Lett. 87, 043503 (2005).

11. S. Rosenblatt, H. Lin, V. Sazonova, et al., Appl. Phys. Lett. 87, 153111 (2005).

12. I. V. Kukushkin, J. H. Smet, V. A. Kovalskii, et al., Phys. Rev. B 72, 161317 (2005).

13. V. M. Muravev, C. Jiang, I. V. Kukushkin, et al., Phys. Rev. B 75, 193307 (2007).

14. M. Lee, M. C. Wanke, and J. L. Reno, Appl. Phys. Lett. 86, 033501 (2005).

15. M. I. Dyakonov and M. S. Shur, IEEE Trans. Electron Devices 43, 380 (1996).

16. I. V. Kukushkin, M. Yu. Akimov, J. H. Smet, et al., Phys. Rev. Lett. 92, 236803 (2004).

17. V. M. Muravev, I. V. Kukushkin, A. L. Parahonskii, et al., JETP Lett. 83, 246 (2006).

18. V. M. Muravev, I. V. Andreev, I. V. Kukushkin, et al., JETP Lett. 87, 577 (2008).

19. V. A. Volkov and S. A. Mikhailov, JETP 94, 217 (1988).

20. I. V. Kukushkin and V. M. Muravev, U.S. Patent Application "Apparatus and Method of Detecting Electromagnetic Radiation."

Translated by R. Tyapaev 